\title{
Spin dephasing in pseudomagnetic fields: susceptibility and geometry
}

\author{
I.V. Tokatly ${ }^{1,2}$ and E.Ya. Sherman ${ }^{3,2}$ \\ ${ }^{1}$ Nano-bio Spectroscopy group and ETSF Scientific Development Centre \\ Dpto. Física de Materiales, Universidad del País Vasco \\ Centro de Física de Materiales CSIC-UPV/EHU-MPC, E-20018 San Sebastian, Spain \\ ${ }^{2}$ IKERBASQUE Basque Foundation for Science, Bilbao, Spain \\ ${ }^{3}$ Department of Physical Chemistry, Universidad del País Vasco UPV-EHU, 48080 Bilbao, Spain \\ E-mail: evgeny.sherman@ehu.eus
}

Received January 4, 2016, published online March 23, 2016

\begin{abstract}
We present a theory of spin dynamics caused by spin-orbit coupling for two-dimensional gases of cold atoms and other quasiparticles with pseudospin 1/2 moving in orbital gauge fields. Our approach is based on the gauge transformation in the form of a SU(2) rotation gauging out the spin-orbit coupling. As a result, the analysis of the spin dynamics is reduced to calculation of the density-related susceptibility of the system without spin-orbit coupling at the wavevector determined by the spin-rotation length. This approach allows one to treat the spin dynamics in terms of the linear response theory for bosonic and fermionic ensembles. We study different regimes of irreversible spin relaxation and coherent spin dynamics in these systems. For bosonic gases the effects of low temperature are crucial due to accumulation of particles in the small-momentum subspace even if the BoseEinstein condensation does not occur due to the system low dimensionality.

PACS: $03.75 . S s$ Degenerate Fermi gases;

05.30.Fk Fermion systems and electron gas;

67.85.-d Ultracold gases, trapped gases.
\end{abstract}

Keywords: spin dynamics, spin-orbit coupling, fermions, bosons.

\section{Introduction}

Recently achieved abilities in producing strong coherent interactions between light and matter led to discovery of a variety of fascinating new phenomena in solids [1] and cold atomic gases $[2,3]$. These phenomena are attributed to the properties of combined quasiparticles arising as a result of such a strong coherent coupling, that is produced by “dressing” of states of real particles or relatively simple quasiparticles (e.g., excitons) by interaction with the optical fields. One of the examples of these quasiparticles is given by polaritons which appear, e.g., as a result of light interaction with the optical interband electron transitions (exciton-polaritons) or with optical lattice vibrations. The latter were predicted by Tolpygo [4] as early as in 1950 and became the first example of such combined states of two quasiparticles, here of optical phonons and photons. The exciton-polaritons based on the interband optical transitions in solids are very light quasiparticles and, as a result of their small effective mass, at achievable concentrations can undergo a Bose-Einstein condensation at relatively high temperatures of the order of $100 \mathrm{~K}$. The Bose-Einstein condensation of much heavier optically-cooled ultracold alkali atoms, with low concentrations (of the order of $10^{14}-10^{15} \mathrm{~cm}^{-3}$ ) occurs at much lower temperatures (of the order of a micro-Kelvin) [2] and can be done in a controllable way, in contrast to the textbook example of the Bose-Einstein condensation of liquid ${ }^{4} \mathrm{He}$.

Many of these novel phenomena reveal the importance of a new quantum degree of freedom determined by the coherent light-matter interaction, that is the quasiparticle pseudospin. It happens that pseudospin $1 / 2$ can be realized for exciton-polaritons in terms of the polarization of the light emitted by them [5] or for coherent exciton gases in terms of spin-orbit coupling for electrons and holes building the excitons [6]. Cold alkali atoms such as ${ }^{87} \mathrm{Rb}$ isotope, even having by themselves bosonic nature with zero total spin formed by the sum of the nuclear and electron 
spins, acquire pseudospin 1/2 (related to the hyperfine structure of atomic levels) due to interaction with the resonant highly coherent laser light. As soon as the pseudospin 1/2 appears, one can produce optically a synthetic spinorbit coupling [7,8] having different forms, usually referred to as the Dresselhaus [9] and Rashba [10,11] ones. We emphasize that the physical origin of these synthetic couplings, being always relativistic in the nature, is completely different from their solid-state “analogues”. Recently, artificial spin-orbit coupling was produced optically in degenerate ultracold atomic Fermi gases such as ${ }^{40} \mathrm{~K}$ and ${ }^{6} \mathrm{Li}[12,13]$, demonstrating the general character if this effect.

The linear-in-the quasiparticle momentum spin-orbit coupling Hamiltonians can be presented as

$$
H_{D}=\alpha_{D}\left(k_{x} \sigma_{x}-k_{y} \sigma_{y}\right)
$$

for the Dresselhaus and

$$
H_{R}=\alpha_{R}\left(k_{x} \sigma_{y}-k_{y} \sigma_{x}\right)
$$

for the Rashba contributions, respectively. Here $k_{i}$ are the Cartesian components of the momentum operator, $\sigma_{i}$ are the Pauli matrices, and $\alpha_{D}$ and $\alpha_{R}$ are the corresponding coupling constants. (We use the units with $\hbar \equiv 1$.) We mention here that initially the Dresselhaus Hamiltonian [9] was obtained for bulk semiconductors without inversion element in their symmetry group, and this coupling was shown to be proportional to $k^{3}$. The linear in- $k$ form of the Dresselhaus coupling is the result of projection of the bulk Hamiltonian onto two-dimensional states localized in the $z$ direction [14].

It is important to mention that the relative strength of spin-orbit coupling compared to the kinetic energy in cold atoms is much larger than that in semiconductors. Indeed, the kinetic energy behaves as $k^{2} / M$, where $M$ is the particle's mass, while the spin-orbit coupling energy increases as $\alpha k$ (where $\alpha$ is a characteristic value of the spin-orbit coupling constant) making the small-momentum systems with large mass more favorable for observation of novel effects of the spin-orbit coupling.

In addition to the synthetic spin-coupling, orbital synthetic gauge fields acting at the motion of particles can optically be produced for cold atoms [15]. These fields, where the orbital effect of the (pseudo)magnetic field produced by the Lorentz force provide a well-known example, qualitatively influence motion of the particles. Through the spin-orbit coupling, these fields determine the collective spin dynamics in the ensembles of particles. Thus, the ability to produce synthetic orbital gauge fields in spin-orbit coupled systems motivates studies of both coherent spin dephasing and irreversible spin relaxation in these systems. This analysis, based in the mapping of the spin dynamics onto the system susceptibility and linear response to an external field, will be presented in this paper.

\section{Spin dynamics and susceptibility for a macroscopic system}

The total Hamiltonian for a macroscopic spin-orbit coupled system which determines the spin dynamics can be expressed using two-component field operators $\tilde{\psi}^{\dagger}$ and $\tilde{\psi}$ as

$$
\hat{H}=\frac{1}{2 M} \int d^{2} r\left\{\tilde{\psi}^{\dagger}(k-A)^{2} \tilde{\psi}\right\}+\hat{H}_{\mathrm{int}}\left[\tilde{\psi}^{\dagger}, \tilde{\psi}\right],
$$

where $r=(x, y)$. This Hamiltonian describes the linear in momentum spin-orbit coupling as a non-Abelian (generally, with nonzero commutator of the Cartesian components $\left[A_{i}, A_{j}\right]$ determined by the $\alpha_{D}$ and $\alpha_{R}$ constants) spin-dependent and momentum-independent gauge field. The interactions between the particles and with an external potential, including either regular confinement or possible disorder, is described by $\hat{H}_{\text {int }}\left[\tilde{\psi}^{\dagger}, \tilde{\psi}\right]$.

We consider here a balanced Rashba-Dresselhaus coupling with $\alpha \equiv \alpha_{D}=\alpha_{R}$, where the single-particle spinorbit coupling Hamiltonian acquires the form

$$
\begin{aligned}
H_{\text {so }} \equiv H_{D}+ & H_{R}=\alpha\left(k_{x}-k_{y}\right)\left(\sigma_{x}+\sigma_{y}\right) \equiv \\
& \equiv \tilde{\alpha}(\mathbf{k} \cdot \mathbf{\kappa})(\boldsymbol{\sigma} \cdot \mathbf{h}),
\end{aligned}
$$

where $\tilde{\alpha}=2 \alpha$, and unit-length vectors are $\boldsymbol{\kappa}=(1,-1,0) / \sqrt{2}$ and $\mathbf{h}=(1,1,0) / \sqrt{2}$. Here the spin-orbit coupling is characterized by a single direction $\mathbf{h}$ of the spin precession [16,17]. This coupling can be gauged away from the Hamiltonian by a coordinate-dependent SU(2) rotation [18-20] in the form

$$
\mathcal{U}=\exp [i \tilde{\alpha} M(\mathbf{r} \cdot \boldsymbol{\kappa})(\boldsymbol{\sigma} \cdot \mathbf{h})]=\exp \left[i \frac{(\mathbf{r} \cdot \mathbf{\kappa})}{2 L_{\text {so }}}(\boldsymbol{\sigma} \cdot \mathbf{h})\right]
$$

The coupling here is characterized by the spin precession length $L_{\text {so }}=1 / 2 M \tilde{\alpha}$, that is the distance the particle should travel to rotate the spin due to the spin-orbit coupling. This rotation, being applied to the uniform spin density distribution [19], produces a nonuniform spin helix [21,22] state. It is interesting to note that both for electrons in semiconductors and for cold atoms the spin rotation length $L_{s o}$ has the value of the order of $10^{-4} \mathrm{~cm}$ although the coupling constants and masses are completely different. Indeed, large masses (of the order of $10^{5} \mathrm{~m}_{0}$, where $m_{0}$ is the free electron mass) and weak couplings ( $\alpha \sim 1 \mathrm{~cm} / \mathrm{s}$ ) for atoms lead to the same order of magnitude result as the light effective masses $\left(\sim 10^{-1} m_{0}\right)$ and stronger couplings $\left(\alpha \sim 10^{6} \mathrm{~cm} / \mathrm{s}\right)$ for the electrons.

For this kind of coupling the spin dynamics can be presented in the form related solely to the spin susceptibility of the system without the spin-orbit coupling [20]. Total spin projection at the $\mathbf{h}=(\mathbf{x}+\mathbf{y}) / \sqrt{2}$ axis remains constant while the perpendicular component evolves with time in a conventional way, including oscillations and relaxa- 
tion. The evolution of the perpendicular to $\mathbf{h}$ component $S_{\perp}(t)$ can be presented in terms of the spin diffusion (here we assume summation over repeated indices):

$$
S_{\perp}^{\sigma_{1}}(\mathbf{r}, t)=\int \mathcal{D}^{\sigma_{1} \sigma_{2}}\left(\mathbf{r}-\mathbf{r}^{\prime}, t\right) S_{\perp}^{\sigma_{2}}\left(\mathbf{r}^{\prime}, 0\right) d^{2} r^{\prime},
$$

where $\mathcal{D}^{\sigma_{1} \sigma_{2}}\left(\mathbf{r}-\mathbf{r}^{\prime}, t\right)$ is the exact "spin diffusion" kernel for spin components $\sigma_{1}$ and $\sigma_{2}$. In the weak interaction and weak disorder-limit this kernel describes the ballistic propagation of particles. It was shown in [20] that in the absence of spin-dependent ordering, its diagonal Fourier components can be presented in the form

$$
\mathcal{D}^{\sigma \sigma}(q, \omega)=\frac{1}{\omega}\left[\frac{\chi_{\sigma \sigma}(q, \omega)}{\chi_{\sigma \sigma}(q, 0)}-1\right],
$$

where $\chi_{\sigma \sigma}(q, \omega)$ is diagonal spin susceptibility in the system without spin-orbit coupling taken at the wavevector $\mathbf{q}=q \boldsymbol{\kappa}$ determined by the spin precession length as $q \equiv 1 / L_{s o}$. Under these conditions, $\chi_{\sigma \sigma}(q, \omega) \equiv \chi(q, \omega) \delta_{\sigma \sigma}$, and the time-dependence $S_{\perp}(t)$ is related to the local density response function $\chi(q, \omega)$ as [20]

$$
S_{\perp}(t)=S_{\perp}(0) \int_{-\infty}^{\infty} \frac{d \omega}{2 \pi i}\left[\frac{\chi(q, \omega)}{\chi(q, 0)}-1\right] \frac{\mathrm{e}^{-i \omega t}}{\omega} .
$$

In the spatially uniform $q \rightarrow 0$ limit, the static spatially uniform susceptibility $\chi(q \rightarrow 0,0)$ is determined by derivative $\partial n / \partial \mu$, where $n$ is the particle concentration and $\mu$ is the chemical potential since it is given by the change in the particle's concentration produced by a spatially smooth external potential.

This equation for spin evolution is general, being valid for any system with spin-orbit coupling (4) and without a spin-dependent magnetism. Therefore, the analysis of the spin dynamics is reduced to the calculation of finitefrequency finite-momentum susceptibility of a system in a magnetic field. For free particles the susceptibility can be presented as a conventional Lindhard function. For particles in gauge field, where closed trajectories are formed, this problem is more complicated as it will be discussed below.

\section{General approaches to susceptibility}

We consider ensembles of particles in a pseudomagnetic field parallel to the $z$ axis imposing a Lorentz force on a particle in the $(x y)$ plane, thus, forming, in the classical mechanics and infinite momentum relaxation time the circular orbital motion or leading to the formation of the set of Landau-Fock states in the quantum regime. Here we present and compare two views on the spin dynamics. One approach is based on the full quantum treatment of the problem. The second one is based on the Boltzmann kinetic equation including the Lorentz force produced by the gauge magnetic fields acting on the particles.

\subsection{Quantum mechanical susceptibility}

We begin with the quantum description of the susceptibility [23] for the Landau-Fock levels. The eigenstates of a particle in a pseudomagnetic field in the Landau gauge are

$$
\psi(x, y)=\frac{1}{\sqrt{l L_{y}}} \exp \left[i k_{y} y\right] \exp \left[-\frac{\left(x-x_{0}\right)^{2}}{2 l^{2}}\right] H_{n}\left(\frac{x-x_{0}}{l}\right),
$$

where $l \sim \sqrt{1 / B}$ is the magnetic oscillator length, $k_{y}$ is the corresponding momentum component, $x_{0}$ is the center of magnetic oscillator position, $H_{n}(z)$ are the properly normalized Hermitian polynomials, and $L_{y}$ is the length of the system in the $y$ direction. The susceptibility has the form of modified Lindhard function and can be presented for unit sample area as the sum over all allowed transitions as

$\chi(q, \omega)=\sum \frac{f\left(\varepsilon_{j \sigma}\right)-f\left(\varepsilon_{j^{\prime} \sigma}\right)}{\omega-\left(\varepsilon_{j^{\prime} \sigma}-\varepsilon_{j \sigma}\right)+i \eta}\left|\left\langle j^{\prime}, k_{y}^{\prime}\left|\hat{n}_{q}\right| j, k_{y}\right\rangle\right|^{2}$,

where each level is characterized by number $j$ and spin projection $\sigma$ and has the energy $\varepsilon_{j \sigma}$ and filling $f\left(\varepsilon_{j \sigma}\right)$, $\hat{n}_{q}$ is the corresponding density operator, and $\eta$ is the Landau level width. As a result, one obtains [23]

$$
\begin{gathered}
\chi(q, \omega)=\frac{\exp \left[-q^{2} l^{2} / 2\right]}{2 \pi l^{2}} \sum_{k=1}^{\infty} \sum_{j}^{\prime} \frac{j !}{(j+k) !}\left(\frac{q^{2} l^{2}}{2}\right)^{k} \times \\
\times\left[L_{j}^{k}\left(\frac{q^{2} l^{2}}{2}\right)\right]^{2}\left[\frac{1}{\omega-k \omega_{c}+i \eta}-\frac{1}{\omega+k \omega_{c}+i \eta}\right] .
\end{gathered}
$$

Here summation over $j$ is taken from $\max \left(0, N_{L}-k\right)$ to $N_{L}$ where $N_{L}$ is the highest occupied Landau level, $L_{j}^{k}$ stands for the Laguerre polynomial, and $\omega_{c}$ is the cyclotron frequency. The susceptibility is a complicated function of momentum and frequency, evidently providing zero susceptibility at zero momentum due the gapped character of the spectrum formed by the orbital motion.

\subsection{Kinetic equation approach}

Here we consider another approach based on the classical kinetic Boltzmann equation in the simple $\tau$-approximation form. From this equation we find susceptibility as a response of a gas in a nonquantizing magnetic field influencing only the orbital motion of the particles to a weak coordinate- and time-dependent perturbation $V_{q}$ at frequency $\omega$ and wavevector $\mathbf{q}$. The knowledge of the susceptibility allows one to obtain the spin evolution with Eq. (8).

The corresponding collision integral has the form

$$
I_{\mathbf{p}}\left[\delta f_{\mathbf{p}}\right]=-\frac{1}{\tau}\left[\delta f_{\mathbf{p}}-\langle\delta f\rangle_{p}\right]
$$

where the mean value is defined by angular averaging as 


$$
\langle\delta f\rangle_{p} \equiv \frac{1}{2 \pi} \int_{0}^{2 \pi} \delta f_{\mathbf{p}} d \varphi
$$

with $\delta f_{\mathbf{p}}$ being the variation in the distribution function and $\varphi$ being the angle between $\mathbf{p}$ and $\mathbf{q}$. The kinetic equation for the distribution functions has the form

$$
\begin{gathered}
i\left(\omega+\frac{i}{\tau}-\frac{p q}{M} \cos \varphi\right) \delta f_{\mathbf{p}}+\omega_{c} \frac{\partial}{\partial \varphi} \delta f_{\mathbf{p}}= \\
=i \frac{p q}{M} \cos \varphi \frac{\partial f_{0}}{\partial \varepsilon} V_{q}-\frac{1}{\tau}\langle\delta f\rangle_{p} .
\end{gathered}
$$

The solution can be presented as

$$
\delta f_{\mathbf{p}}=V_{q} \frac{\partial f_{0}(\varepsilon)}{\partial \varepsilon} \psi_{q}(p, \varphi)
$$

where function $\psi_{q}(p, \varphi)$ is determined by self-consistent equation

$$
\begin{gathered}
i\left(\omega+\frac{i}{\tau}-\frac{p q}{M} \cos \varphi\right) \psi_{q}(p, \varphi)-\omega_{c} \frac{\partial}{\partial \varphi} \psi_{q}(p, \varphi)= \\
=i \frac{p q}{M} \cos \varphi+\frac{i}{\tau}\left\langle\psi_{q}(p, \varphi)\right\rangle .
\end{gathered}
$$

The susceptibility is obtained by integrating over the momenta of the particles:

$$
\chi(q, \omega)=\int \psi_{q}(p, \varphi) \frac{\partial f_{0}(\varepsilon)}{\partial \varepsilon} \frac{d^{2} p}{(2 \pi)^{2}} .
$$

Using definition of Bessel function of the $n$th order

$$
J_{n}(z)=\frac{1}{2 \pi} \int_{0}^{2 \pi} \exp (i n z \cos \phi) d \phi,
$$

and introducing functions

$$
\begin{aligned}
& \mathcal{I}_{0}^{[p]}(q, \omega) \equiv \sum_{n=-\infty}^{n=\infty} \frac{n \omega_{c}}{\omega+i / \tau-n \omega_{c}} J_{n}^{2}\left(\frac{p q}{M \omega_{c}}\right), \\
& \mathcal{I}_{1}^{[p]}(q, \omega) \equiv \sum_{n=-\infty}^{n=\infty} \frac{\omega-n \omega_{c}}{\omega+i / \tau-n \omega_{c}} J_{n}^{2}\left(\frac{p q}{M \omega_{c}}\right)
\end{aligned}
$$

we obtain with some algebra from Eq. (16) for the susceptibility

$$
\chi(q, \omega)=\int_{0}^{\infty} \frac{\partial f_{0}(\varepsilon)}{\partial \varepsilon} \frac{\mathcal{I}_{0}^{[p]}(q, \omega)}{\mathcal{I}_{1}^{[p]}(q, \omega)} v d \varepsilon,
$$

where $v=M / 2 \pi$ is the energy-independent density of states per single spin component in a two-dimensional gas. The factor $p q / M \omega_{c}$ has simple geometrical meaning. Taking into account that $p / M \omega_{c}=R_{c}$, where $R_{c}$ is the cyclotron radius, we see that the dynamics is determined by a product $q R_{c}$, that is by the ratio $R_{c} / L_{s o}$. We will use this fact in the analysis of the spin evolution.
The above equations are exact in the $\tau$-approximation being particle statistics-independent. They determine full frequency- and momentum-dependent susceptibility of a macroscopic system in a nonquantizing magnetic field and resulting spin evolution. The value of $\tau$ can have several origins. First origin is related to the disorder, which always presents in solids and can be produced in cold atomic gases by random optical fields. This disorder leads to relaxation both in the total momentum and in the spin. However, in the context of spin dynamics, the relaxation can be caused by interparticle collisions, leading to the spin drag, not contributing into the total momentum relaxation $[24,25]$. Since these interparticle collisions are strongly temperature-dependent, they can lead to a strong modification of the spin relaxation with the temperature, which can be qualitatively different for Fermi and Bose gases.

\section{Spin dynamics: irreversible and reversible}

Here we apply the susceptibility-based approach to several regimes such as the irreversible spin relaxation due to collisions and reversible dephasing in clean gases. This approach is valid for electrons in two-dimensional semiconductors and for cold of two-dimensional fermionic and bosonic gases. We will see the importance of the $R_{c} / L_{s o}$ ratio in these regimes.

\subsection{Diffusion limit and irreversible relaxation}

We begin with the irreversible spin relaxation arising due to the collisions with the characteristic rate of $1 / \tau$. Here we explore the limit of low-frequencies and, correspondingly, the long-time behavior of spin density corresponding to $\omega \ll 1 / \tau$, and $\omega \ll \omega_{c}$. At long times, after many scatterings, the particle motion becomes diffusion with the displacement $\left\langle r^{2}(t)\right\rangle \sim D t$, where $D$ is the diffusion coefficient. Here the susceptibility is determined by the diffusion pole [26] in the system response, common for the Bose and Fermi statistics, presented in the form

$$
\mathcal{D}(\omega, q)=\frac{1}{D(B) q^{2}-i \omega} .
$$

By making expansion with small parameter $\omega \tau$ in Eqs. (19) and (20) and using the small $z$ behavior $J_{n}(z) \sim z^{n}$, we obtain that only the $n=1$ and $n=0$ terms contribute into the susceptibility in the nominator and denominator, respectively, and the field-dependent diffusion coefficient becomes

$$
D(B)=\frac{D(0)}{1+\omega_{c}^{2} \tau^{2}} .
$$

Here $D(0)$ is the diffusion coefficient in the absence of magnetic field, equal, e.g., $v_{F}^{2} \tau / 2$ for highly degenerate Fermi gas with the Fermi velocity $v_{F}$, and $D(B)$ decreases as a result of the cyclotron motion, making in the strong- 
field limit $\omega_{c}^{2} \tau^{2} \gg 1$ the cyclotron radius much smaller than the particle free part. Integrating over $\omega$ in Eq. (8), we obtain the conventional exponential Dyakonov-Perel' spin relaxation [27] in the form of Refs. 28, 29:

$$
S_{\perp}(t)=S_{\perp}(0) \exp \left(-D(B) q^{2} t\right)
$$

as expected. The spin relaxation rate $D(B) q^{2}$ is proportional to the momentum relaxation time included in $D(B)$ and square of the spin-orbit coupling constant in the wavevector $q$ and suppressed by the circular motion. This formula can be seen as a long, taking time of the order of $L_{s o}^{2} / D(B)$, diffusive smearing of the particle's density variation on the timescale of the order of the time necessary for the particle, either a free one (at $B=0$ ) or performing the cyclotron motion, to diffuse through the spatial domain of the characteristic spin-flip $L_{s o}$ size. Thus, the Dyakonov-Perel' spin relaxation is directly mapped onto the particle diffusion in the real space.

\subsection{Clean limit: geometry and reversible dephasing}

Here we consider clean systems (or short-term behavior), where the scattering plays a negligible role, and the dephasing becomes reversible. Using integral representation of the Bessel function, one can obtain that for the element of the distribution function with momentum $p$ one can present the time-dependent spin component as

$$
\frac{s_{p, q}(t)}{s_{p, q}(0)}=J_{0}\left(\frac{2 p q}{M \omega_{c}} \sin \frac{\omega_{c} t}{2}\right) .
$$

This expression has a simple physical and geometrical meaning, corresponding to the dephasing of particles' spins on circular path of the momentum $p$-dependent radius of $R_{c}(p)$. All particles start to move from the origin and return to it at the time of $2 \pi / \omega_{c}$. Spin precession angle is proportional the displacement along the $(\mathbf{x}-\mathbf{y}) / \sqrt{2}$ axis, and summation of all spins leads to the above spin dependence. This process is illustrated in Fig. 1. The resulting spin dephasing is given by averaging over the ensemble as

$$
\frac{S_{\perp}(t)}{S_{\perp}(0)}=\frac{\mathcal{J}(t)}{\mathcal{J}(0)},
$$

where

$$
\mathcal{J}(t)=\int_{0}^{\infty} \frac{\partial f_{0}(\varepsilon)}{\partial \varepsilon} J_{0}\left(2 R_{c}(\varepsilon) q \sin \frac{\omega_{c} t}{2}\right) v d \varepsilon,
$$

and $p=\sqrt{2 M \varepsilon}$.

Note that

$$
\mathcal{J}(0)=\int_{0}^{\infty} \frac{\partial f_{0}(\varepsilon)}{\partial \varepsilon} \nu d \varepsilon=-v f_{0}(0)=-\frac{\partial n}{\partial \mu}
$$

corresponds to the static uniform susceptibility of the system.

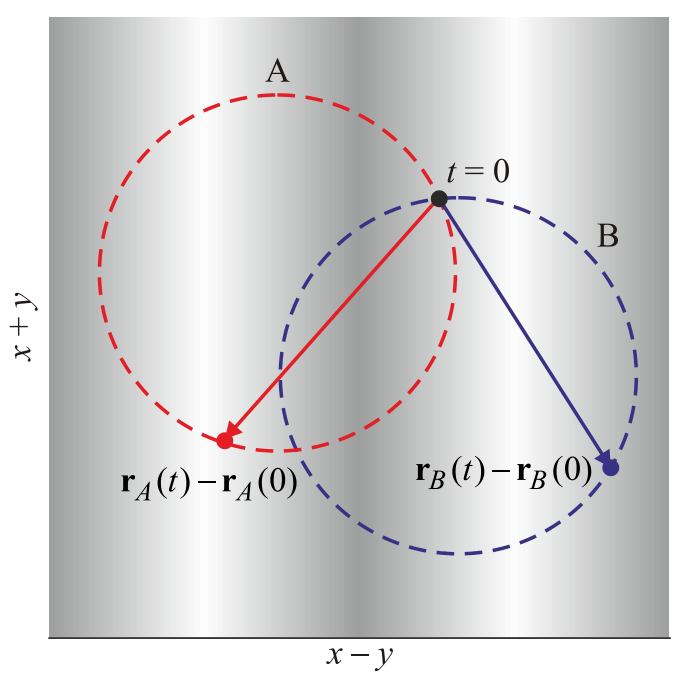

Fig. 1. (Color online) Two different circular path (A, B) in a magnetic field. Arrows show the displacement of the particles at time $t$. Although the displacements of particle at time $t$ are equal, $\left|\mathbf{r}_{A}(t)-\mathbf{r}_{A}(0)\right|=\left|\mathbf{r}_{B}(t)-\mathbf{r}_{B}(0)\right|$, their projections at the $(\mathbf{x}-\mathbf{y}) / \sqrt{2}$ axis are different, resulting in different spin precession angles. The dephasing occurs when the difference in the displacements along the $(\mathbf{x}-\mathbf{y}) / \sqrt{2}$ axis is of the order of $L_{S O}$, corresponding to the spacial scale of the periodic inhomogeneity in the background of the figure.

In addition, we mention what happens in the weak-field limit where $R_{c} \gg L_{s o}$. Here, we can assume $\omega_{c} t \ll 1$ and obtain

$$
\mathcal{J}(t)=\int_{0}^{\infty} \frac{\partial f_{0}(\varepsilon)}{\partial \varepsilon} J_{0}\left(\frac{p q}{M} t\right) \nu d \varepsilon
$$

that is oscillating behavior with slowly $(\sim 1 / \sqrt{t})$ decreasing amplitude, corresponding to spin dephasing in the absence of magnetic field. This dephasing occurs on the time scale of the reversible smearing of the density fluctuation of the order of $L_{s o} / v$, where $v$ is the typical the particle velocity.

\section{Coherent spin dephasing: the role of statistics}

In this section we concentrate on the coherent reversible spin dephasing in cold two-dimensional fermionic and bosonic gases and show the qualitative role of the statistics in the spin dynamics.

\subsection{Cold fermions}

Here we consider spin dephasing of highly degenerate clean Fermi gas described by distribution function:

$$
f=\frac{1}{\exp [(\varepsilon-\mu) / T]+1} .
$$




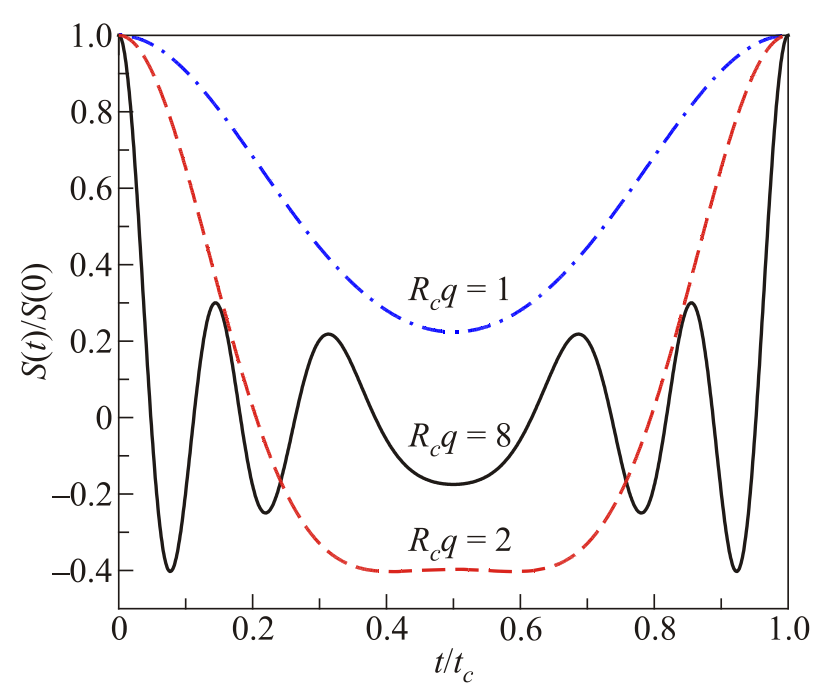

Fig. 2. (Color online) Spin dephasing for fermions. This plot presents functions $J_{0}\left(2 R_{C} q \sin \left(\omega_{C} t / 2\right)\right)$ for different values of $R_{C} q$ as marked near the plots, where $t_{C} \equiv 2 \pi / \omega_{C}$. Same result was obtained in Ref. 30 by direct solution of the kinetic equation in the presence of spin-orbit coupling.

Condition of low temperature can be formulated as $T \ll \mu=E_{F}$, and all the physics is related to the Fermisurface properties characterized by Fermi momentum $p_{F}=\sqrt{2 M E_{F}}$. We mention that the zero-frequency limit of the susceptibility is given by the density of states at the Fermi level $v$, that is by the energy-independent particle mass $M$. The condition of classical motion here is formulated as $E_{F} \gg \omega_{C}$.

In the collisionless limit we obtain oscillating timedependence (Fig. 2)

$$
S_{\perp}(t)=S_{\perp}(0) J_{0}\left(\frac{2 v_{F} q}{\omega_{C}} \sin \frac{\omega_{C} t}{2}\right) .
$$

This can be understood as the reversible dephasing due to the regular circular motion and obtained on simple physical grounds of trajectories starting at the same point at $t=0$, as can be seen in Fig. 1 . All particles participating in the dephasing have the same cyclotron radii $v_{F} / \omega_{C}$ and, therefore, move the same distance at given time $t$. This means that the dephasing strongly depends on the cyclotron radius common for all the trajectories of the particles with momentum $p_{F}$. Equation (31) clearly shows revivals in the spin after each cyclotron period $2 \pi / \omega_{C}$. If finite collision rate is taken into account, amplitude of the revival peaks decays exponentially as

$$
S_{\perp}(t)=S_{\perp}(0) J_{0}\left(\frac{2 v_{F} q}{\omega_{c}} \sin \frac{\omega_{c} t}{2}\right) \exp (-t / \tau) .
$$

We mention that for the degenerate Fermi gas $\tau$ is proportional to $T^{-2}$, and, therefore, in this limit occurs reversible ballistic, almost temperature-independent, spin dynamics, solely determined by the $R_{C} / L_{S O}$ ratio.

\subsection{Cold bosons}

The Bose statistics, where the equilibrium distribution function has the form

$$
f_{0}(\varepsilon)=\frac{1}{\exp [(\varepsilon-\mu) / T]-1},
$$

leads to a qualitatively different spin dynamics. Since there is no Bose-Einstein condensation of free two-dimensional bosons, we assume that at any temperature $\mu<0$, and the condition of high degeneracy can be formulated as $|\mu| \ll T$. This allows us to present the distribution function in the form

$$
f_{0}(\varepsilon)=\frac{T}{\varepsilon+|\mu|}
$$

describing the particles accumulation at low energies in the absence of Bose-Einstein condensation at the energy interval $\varepsilon \ll T$. Temperature behavior of chemical potential at low $T$ is given by: $|\mu|=T \exp \left(-T_{g} / T\right) \ll T$, where $T_{g}=n / M$ is the degeneracy temperature where the classical gas becomes a quantum one. Although the chemical potential does not drop to zero at any finite $T$, it is exponentially small in a large interval of temperatures $T \ll T_{g}$. The condition of classical motion in the magnetic field is $T \gg \omega_{c}$.

To describe spin dynamics we introduce characteristic cyclotron radius $R_{C}^{[T]} \equiv \sqrt{T / M} / \omega_{C}$ and note that it satisfies condition $R_{C}^{[T]} \gg R_{C}^{\min } \equiv 1 / \sqrt{M \omega_{C}}$. The resulting evolution of the spin in the Bose gas presented in Fig. 3

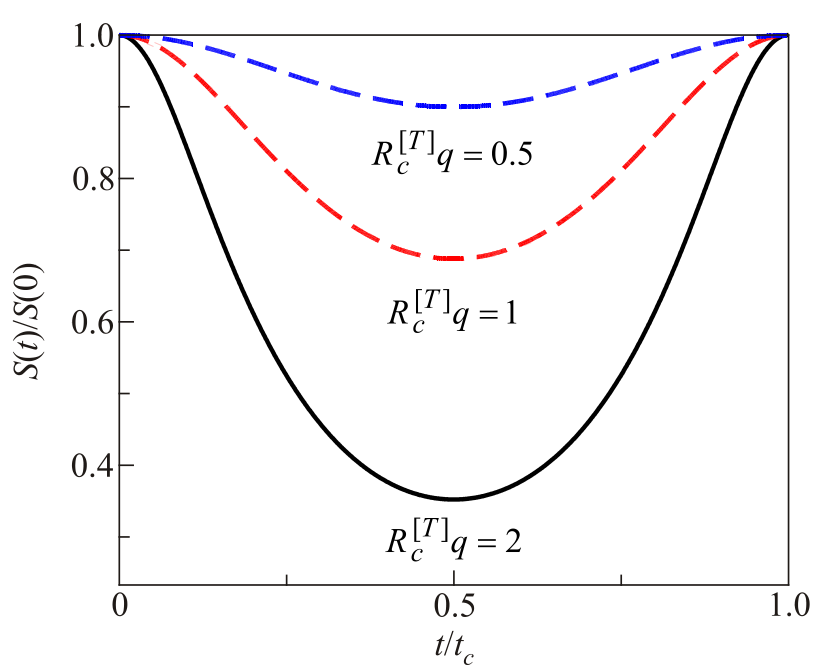

Fig. 3. (Color online) Reversible spin dephasing for bosons. The plot presents time-dependence of spin for different parameters $R_{C}^{[T]} q$. The values of chemical potential are taken as $\mu=-0.1 T$ for a highly degenerate Bose gas. Note that, in contrast to the Fermi gas in Fig. 2, the $S(t) / S(0)$ dependence does not show oscillations since the width of thermal distribution in the cyclotron radii $R_{C} \sim \sqrt{T / M} / \omega_{C}$ is of the order of its mean value. As a result, the spin dephasing and revivals occur without oscillations. 
strongly depends on the temperature and does not show oscillations due to a broad distribution of the cyclotron radii $R_{C}(\varepsilon)$, produced by a broad energy distribution. For any temperature, we see general revivals at time $t_{c}$, when all the particles return to their initial positions. At $T>M\left(L_{s o} \omega_{c}\right)^{2}$, total spin decreases almost to zero at a short time $t_{0} \sim L_{s o} \sqrt{M / T}$ and then restores after the cyclotron period. If $R_{c}^{\min }<L_{s o}$, the effect of spin dephasing becomes small as the gas is cooled down. Otherwise, the dephasing is strong in the entire semiclassical region of temperatures.

\section{Conclusions}

We presented a theory of spin dynamics in Fermi and Bose gases based on the SU(2) coordinate-dependent spin rotation, which allowed us to gauge out the spin-orbit coupling and to reduce the analysis to the properties of susceptibility of a system without spin-orbit coupling. We obtained that the spin dynamics can be expressed in terms of the ratio of characteristic cyclotron radius determined by the system parameters such as the magnetic field, concentration, and the temperature to the spin-precession length determined solely by the fundamental parameters such as the particle mass and the spin-orbit coupling constant. We found the Dyakonov-Perel' and reversible dephasing regimes of spin dynamics. In addition, we have clearly seen that the spin dynamics of the Bose gas is suppressed at low temperatures as the mean energy of particle decreases even if the Bose-Einstein condensation does not occur.

\section{Acknowledgment}

We acknowledges funding by the Spanish MEC (FIS2010-21282-C02-01 and FIS2012-36673-C03-01) and "Grupos Consolidados UPV/EHU del Gobierno Vasco" (IT-319-07 and IT-472-10). The contribution of EYS was, in addition, supported by the University of Basque Country UPV/EHU under program UFI 11/55.

1. H. Deng, H. Haug, and Y. Yamamoto, Rev. Mod. Phys. 82, 1489 (2010).

2. W. Ketterle, Rev. Mod. Phys. 74, 1131 (2002).

3. Y.-J. Lin, K. Jimenez-Garcia, and I.B. Spielman, Nature 471, 83 (2011).

4. K.B. Tolpygo, Zh. Eksp. Teor. Fiz. 20, 497 (1950).

5. I.A. Shelykh, A.V. Kavokin, Y.G. Rubo, T.C.H. Liew, and G. Malpuech, Semicond. Sci. Technol. 25, 013001 (2010).

6. A.A. High, A.T. Hammack, J.R. Leonard, Sen Yang, L.V. Butov, T. Ostatnick'y, M. Vladimirova, A.V. Kavokin,
T.C.H. Liew, K.L. Campman, and A.C. Gossard, Phys. Rev. Lett. 110, 246403 (2013).

7. H. Zhai, Int. J. Mod. Phys. B 26, 1230001 (2012).

8. V. Galitski and I.B. Spielman, Nature 494, 49 (2013).

9. G. Dresselhaus, Phys. Rev. 100, 580 (1955).

10. E.I. Rashba, Soviet Physics-Solid State 2, 1109 (1960).

11. Yu.A. Bychkov and E.I. Rashba, JETP Lett. 39, 79 (1984).

12. P. Wang, Z.-Q. Yu, Z. Fu, J. Miao, L. Huang, S. Chai, H. Zhai, and J. Zhang, Phys. Rev. Lett. 109, 095301 (2012).

13. L.W. Cheuk, A.T. Sommer, Z. Hadzibabic, T. Yefsah, W.S. Bakr, and M.W. Zwierlein, Phys. Rev. Lett. 109, 095302 (2012).

14. M.I. Dyakonov and V.Yu. Kachorovskii, Fiz. Tekh. Poluprovodn. 20, 178 (1986) [Sov. Phys. Semicond. 20, 110 (1986)].

15. N. Goldman, G. Juzeliūnas, P. Öhberg, and I.B. Spielman, Rep. Progr. Phys. 77, 126401 (2014).

16. N.S. Averkiev and L.E. Golub, Phys. Rev. B 60, 15582 (1999).

17. J. Schliemann, J.C. Egues, and D. Loss, Phys. Rev. Lett. 90, 146801 (2003).

18. For a general linear in $k$ spin-orbit coupling, the gauge transformation was proposed by I.L. Aleiner and V.I. Fal'ko, Phys. Rev. Lett. 87, 256801 (2001). Although it does not gauge the coupling completely out, it allows to simplify its consideration in terms of perturbation theory.

19. I.V. Tokatly and E.Ya. Sherman, Ann. Phys. 325, 1104 (2010).

20. I.V. Tokatly and E.Ya. Sherman, Phys. Rev. B 82, 161305 (2010).

21. B.A. Bernevig, J. Orenstein, and S.-C. Zhang, Phys. Rev. Lett. 97, 236601 (2006).

22. J.D. Koralek, C.P. Weber, J. Orenstein, B.A. Bernevig, Shou-Cheng Zhang, S. Mack, and D.D. Awschalom, Nature 458, 610 (2009).

23. G. Giuliani and G. Vignale, Quantum Theory of the Electron Liquid, Cambridge University Press (2005).

24. M.M. Glazov and E.L. Ivchenko, Pisma Zh. Eksp. Teor. Fiz. 75, 476 (2002) [JETP Lett. 75, 403 (2002)]; Sov. Phys. JETP 99, 1279 (2004).

25. M. Polini and G. Vignale, Phys. Rev. Lett. 98, 266403 (2007).

26. D. Forster, Hydrodynamic Fluctuations, Broken Symmetry, and Correlation Functions, Benjamin, New York (1975).

27. M.I. Dyakonov and V.I. Perel', Sov. Phys. Solid State 13, 3023 (1972).

28. E.L. Ivchenko, Fiz. Tverd. Tela (Leningrad) 15, 1566 (1973) [Sov. Phys. Solid State 15, 1048 (1973)].

29. The Greens-function formalism for this problem was proposed in A.A. Burkov and L. Balents, Phys. Rev. B 69, 245312 (2004).

30. M.M. Glazov, Solid State Commun. 142, 531 (2007). 